\title{
TMD parton densities in associated real and virtual photon and jet production at LHC
}

\author{
A.V. Lipatov ${ }^{1,2}$, N.P. Zotov ${ }^{1}$ \\ September 24, 2014 \\ ${ }^{1}$ Skobeltsyn Institute of Nuclear Physics, Lomonosov Moscow State University, 119991 \\ Moscow, Russia \\ ${ }^{2}$ Joint Institute for Nuclear Research, 141980 Dubna, Moscow Region, Russia
}

\begin{abstract}
We study the associated production of real (isolated) or virtual photons (with their subsequent leptonic decay) and hadronic jets in proton-proton collisions at the LHC using the $k_{T}$-factorization approach of QCD. The consideration is based on the off-shell quarkgluon QCD Compton scattering subprocesses. In the case of virtual photon production, the contributions from $Z$ boson exchange as well as $\gamma^{*}-Z$ interference with the full spin correlations are included. The transverse momentum dependent (TMD) quark and gluon densities in a proton are determined from the Kimber-Martin-Ryskin prescription or Catani-Ciafoloni-Fiorani-Marchesini (CCFM) equation. In the latter, we restricted to the case where the gluon-to-quark splitting occurs at the last evolution step and calculate the sea quark density as a convolution of the CCFM-evolved gluon distribution and the TMD gluon-to-quark splitting function. Our numerical predictions are compared with the recent experimental data taken by the ATLAS Collaboration. We discuss the theoretical uncertainties of our calculations and argue that further studies are capable of constraining the TMD parton densities in a proton.
\end{abstract}

PACS number(s): 12.38.-t, 12.15.Ji 
Recently, the ATLAS Collaboration has reported data [1,2] on the associated direct photon 1 and hadronic jet production in proton-proton collisions at the LHC. The data on the associated production of lepton pair and jets have been presented also [3]. At present, both these processes are subjects of intense studies. The theoretical and experimental investigations of direct photon production provide a probe of the hard subprocess dynamics since the produced photons are almost insensitive to the effects of final-state hadronization. The corresponding total and differential cross sections are strongly sensitive to the parton (quark and gluon) content of a proton since, at leading order, the direct photons can be produced mainly via quark-gluon Compton scattering or quark-antiquark annihilation. Moreover, such events provide one of the main backgrounds in searches of Higgs bosons decaying to a photon pair. Dilepton production, where final leptons originate from the decay of virtual photon or intermediate $Z$ boson, has a large cross section and clean signature in the detectors and therefore it is used for monitoring the collider luminosity and calibration of detectors. It is an important reference process for measurements of electroweak boson properties and provides a major source of background to a number of processes such as Higgs, $t \bar{t}$ pair, di-boson or $W^{\prime}$ and $Z^{\prime}$ bosons production (and other processes beyond the Standard Model) studied at hadron colliders.

It was claimed [3] that recent ATLAS data on the associated dilepton and hadronic jet production can be reasonably well described by the next-to-leading perturbative QCD predictions (NLO pQCD) computed using the BLACKHAT program [4]. The NLO pQCD calculations [5] (the JeTPHox package) provide also a reasonably good description of the ATLAS data [1,2] on the associated direct photon and jet production, except for the case of azimuthal opening angle between the produced photon and jet. Additionally, it was demonstrated [1] that the theoretical predictions [5] overestimate the measured cross sections at small photon transverse energy $E_{T}^{\gamma}<45 \mathrm{GeV}$.

An alternative description can be achieved within the framework of the $k_{T}$-factorization QCD approach [6, 7]. This approach is based on the Balitsky-Fadin-Kuraev-Lipatov (BFKL) [8] or the Ciafaloni-Catani-Fiorani-Marchesini (CCFM) [9] gluon dynamics and provides solid theoretical grounds for the effects of initial gluon radiation and intrinsic parton transverse momentum 2 . The basic dynamical quantities of the $k_{T}$-factorization formalism are the parton distributions unintegrated over the parton transverse momentum $k_{T}$, or transverse momentum dependent (TMD) parton distributions. At present, these quantities are subject of intense studies, and various theoretical approaches to investigate them have been proposed [11 15]. Nevertheless, most of phenomenological applications take only gluon and valence quark contributions into account (see, for example, [16-20]). Such approach is a reasonable approximation (based on the dominance of spin-1 exchange processes at high energies) for the production processes coupled to the gluons. But, to correctly treat the final states associated with the quark-initiated processes, it is necessary to go beyond this approximation and take into account subleading effects connected, in particular, with the TMD sea quark distribution. First attempts to address this issue have been performed in [21 24], where the TMD sea quark density has been derived from the TMD gluon distribution via splitting probabilities to lowest order of perturbative theory, neglecting any transverse momentum dependence in the gluon-to-quark branching. Recently, the formulation of the TMD sea quark density which incorporates the effects of the TMD gluon-to-quark splitting function [25] have been proposed [26], where the TMD gluon-to-quark splitting function contains all single logarithmic small- $x$ corrections to sea quark evolution for any order of perturbation theory. The proposed formulation has been implemented in a Monte Carlo event generator CASCADE [27], and the specific

\footnotetext{
${ }^{1}$ Usually the photons are called direct if they are coupled to the interacting quarks.

${ }^{2} \mathrm{~A}$ detailed description and discussion of the $k_{T}$-factorization formalism can be found in [10].
} 
kinematical effects from initial state parton transverse momentum on the forward $Z$ boson spectrum have been studied [28]. First phenomenological application of the developed formalism [26, 28] to the analysis of experimental data was made in 29], where the inlcusive Drell-Yan lepton pair production at the LHC has been considered. In the present note we extend this previous investigation by including into the consideration the processes of associated production of direct photons or lepton pairs and hadronic jets at the LHC. As it was mentioned above, both these processes offer high sensitivity to the sea quark evolution in a proton at moderate and high scales (up to $\mu^{2} \sim m_{Z}^{2}$ ).

Let us start from a short review of calculation steps. Our consideration is based on the off-shell quark-gluon QCD Compton-like scattering subprocesses: 3 :

$$
\begin{gathered}
q\left(k_{1}\right)+g^{*}\left(k_{2}\right) \rightarrow \gamma\left(p_{1}\right)+q\left(p_{2}\right), \\
q\left(k_{1}\right)+g^{*}\left(k_{2}\right) \rightarrow Z / \gamma^{*}+q \rightarrow l^{+}\left(p_{1}\right)+l^{-}\left(p_{2}\right)+q\left(p_{3}\right),
\end{gathered}
$$

where the four-momenta of all corresponding particles are given in the parentheses. Since we are interested in the events containing the jets in final state, using the subprocess (2) istead of simple quark-antiquark annihilation (which has been applied previously [29] to the inclusive Drell-Yan production case) is more suitable (see discussion below). Also, we will neglect the virtualities of initial quarks (but not gluons) in production amplitudes of subprocesses (1) and (2) as compared to the quite large hard scale $\mu^{2}$ of such events. Note that contributions from the quark-antiquark annihilation are effectively taken into account in our consideration due to initial state gluon radiation. It is in contrast with collinear QCD factorization where these contributions have to be taken into account separately.

The gauge-invariant off-shell production amplitudes squared of subprocesses (1) and (2) have been calculated in [31] and [32, respectively. These calculations are rather straightforward. We only mention that, in according to the $k_{T}$-factorization prescription [6,7], the summation over the incoming off-shell gluon polarizations is carried out with $\sum \epsilon^{\mu} \epsilon^{* \nu}=$ $\mathbf{k}_{2 T}^{\mu} \mathbf{k}_{2 T}^{\nu} / \mathbf{k}_{2 T}^{2}$, where $\mathbf{k}_{2 T}$ is the gluon transverse momentum, and $k_{2}^{2}=-\mathbf{k}_{2 T}^{2} \neq 0$. In all other respects our calculations follow the standard Feynman rules.

According to the $k_{T}$-factorization approach, to calculate the cross section of processes under consideration one should convolute corresponding off-shell partonic cross sections with the TMD parton densities in a proton. Our master formulas read:

$$
\begin{gathered}
\sigma(p p \rightarrow \gamma+\text { jet })=\sum_{q} \int \frac{1}{16 \pi\left(x_{1} x_{2} s\right)^{2}}\left|\overline{\mathcal{M}}\left(q g^{*} \rightarrow \gamma q\right)\right|^{2} \times \\
\times f_{q}\left(x_{1}, \mathbf{k}_{1 T}^{2}, \mu^{2}\right) f_{g}\left(x_{2}, \mathbf{k}_{2 T}^{2}, \mu^{2}\right) d \mathbf{p}_{1 T}^{2} d \mathbf{k}_{1 T}^{2} d \mathbf{k}_{2 T}^{2} d y_{1} d y_{2} \frac{d \phi_{1}}{2 \pi} \frac{d \phi_{2}}{2 \pi} \\
\sigma\left(p p \rightarrow l^{+} l^{-}+\text {jet }\right)=\sum_{q} \int \frac{1}{256 \pi^{3}\left(x_{1} x_{2} s\right)^{2}}\left|\overline{\mathcal{M}}\left(q g^{*} \rightarrow Z / \gamma^{*} q \rightarrow l^{+} l^{-} q\right)\right|^{2} \times \\
\times f_{q}\left(x_{1}, \mathbf{k}_{1 T}^{2}, \mu^{2}\right) f_{g}\left(x_{2}, \mathbf{k}_{2 T}^{2}, \mu^{2}\right) d \mathbf{p}_{1 T}^{2} d \mathbf{p}_{2 T}^{2} d \mathbf{k}_{1 T}^{2} d \mathbf{k}_{2 T}^{2} d y_{1} d y_{2} d y_{3} \frac{d \phi_{1}}{2 \pi} \frac{d \phi_{2}}{2 \pi} \frac{d \psi_{1}}{2 \pi} \frac{d \psi_{2}}{2 \pi},
\end{gathered}
$$

where $f_{q}\left(x, \mathbf{k}_{T}^{2}, \mu^{2}\right)$ and $f_{g}\left(x, \mathbf{k}_{T}^{2}, \mu^{2}\right)$ are the TMD quark and gluon densities in a proton, $s$ is the total energy, $\mathbf{p}_{1 T}, \mathbf{p}_{2 T}, \psi_{1}, \psi_{2}, y_{1}, y_{2}$ and $y_{3}$ are the transverse momenta, azimuthal angles and center-of-mass rapidities of produced particles, and $\phi_{1}$ and $\phi_{2}$ are the azimuthal angles of the incoming partons having the non-zero transverse momenta $\mathbf{k}_{1 T}$ and $\mathbf{k}_{2 T}$ and fractions $x_{1}$ and $x_{2}$ of the longitudinal momenta of the colliding protons. If we average

\footnotetext{
${ }^{3}$ We will neglect the contributions from the so-called fragmentation mechanisms in the case of direct photon production. It is because after applying the isolation cut (see [1,2]) these contributions amount only to about $10 \%$ of the visible cross section. The isolation requirement and additional conditions which preserve our calculations from divergences have been specially discussed in [30].
} 
these expressions over $\phi_{1}$ and $\phi_{2}$ and take the limit $\left|\mathbf{k}_{1 T}\right| \rightarrow 0$ and $\left|\mathbf{k}_{2 T}\right| \rightarrow 0$, then we recover the corresponding formulas of the collinear QCD factorization.

To calculate the TMD parton densities in a proton we follow the approach [26] based on the CCFM equation. The CCFM parton shower describes only the emission of gluons, while real quark emissions are left aside. It implies that this equation describes only the distinct evolution of TMD gluon and valence quarks, while the non-diagonal transitions between quarks and gluons are absent. The TMD gluon and valence quark distributions have been obtained from the numerical solutions of the CCFM equation in [17, 33]. To calculate the TMD sea quark density we apply the approximation where the sea quarks occur in the last gluon-to-quark splitting. At the next-to-leading logarithmic accuracy $\alpha_{s}\left(\alpha_{s} \ln x\right)^{n}$ the TMD sea quark distribution can be written as follows [26]:

$$
f_{q}^{(\text {sea })}\left(x, \mathbf{q}_{T}^{2}, \mu^{2}\right)=\int_{x}^{1} \frac{d z}{z} \int d \mathbf{k}_{T}^{2} \frac{1}{\Delta^{2}} \frac{\alpha_{s}}{2 \pi} P_{q g}\left(z, \mathbf{k}_{T}^{2}, \Delta^{2}\right) f_{g}\left(x / z, \mathbf{k}_{T}^{2}, \bar{\mu}^{2}\right),
$$

where $z$ is the fraction of the gluon light cone momentum carried out by the quark, $\mathbf{q}_{T}$ and $\mathbf{k}_{T}$ are the sea quark and gluon transverse momenta, $z$ is the fraction of the gluon light cone momentum carried out by the quark, and $\boldsymbol{\Delta}=\mathbf{q}_{T}-z \mathbf{k}_{T}$. The sea quark evolution is driven 4 by the off-shell gluon-to-quark splitting function $P_{q g}\left(z, \mathbf{k}_{T}^{2}, \Delta^{2}\right)$ [25]:

$$
P_{q g}\left(z, \mathbf{k}_{T}^{2}, \Delta^{2}\right)=T_{R}\left(\frac{\Delta^{2}}{\Delta^{2}+z(1-z) \mathbf{k}_{T}^{2}}\right)^{2}\left[(1-z)^{2}+z^{2}+4 z^{2}(1-z)^{2} \frac{\mathbf{k}_{T}^{2}}{\Delta^{2}}\right],
$$

with $T_{R}=1 / 2$. The splitting function $P_{q g}\left(z, \mathbf{k}_{T}^{2}, \Delta^{2}\right)$ has been obtained by generalizing to finite transverse momenta, in the high-energy region, the two-particle irreducible kernel expansion [34]. Although evaluated off-shell, this splitting function is universal: it takes into account the small- $x$ enhanced transverse momentum dependence up to all orders in the strong coupling, and reduces to the conventional splitting function at lowest order for $\left|\mathbf{k}_{T}\right| \rightarrow 0$. The scale $\bar{\mu}^{2}$ is defined [26] from the angular ordering condition which is natural from the point of view of the CCFM evolution: $\bar{\mu}^{2}=\Delta^{2} /(1-z)^{2}+\mathbf{k}_{T}^{2} /(1-z)$. To precise, in (5) we have used A0 gluon [33].

An alternative way to calculate the TMD parton densities in a proton is the KimberMartin-Ryskin (KMR) approach [35], which is a formalism to construct the TMD parton distributions from the known collinear ones. The key assumption of the KMR approach is that the $k_{T}$-dependence of the TMD parton densities enters at the last evolution step, and the conventional Dokshitzer-Gribov-Lipatov-Altarelli-Parisi (DGLAP) evolution equations [36] can be used up to this step. The TMD parton densities calculated using both these approaches have been compared in [29]. Below we will test them numerically 5 .

Other essential parameters were taken as follows: renormalization and factorization scales $\mu_{R}=\mu_{F}=\xi E_{T}^{\gamma}$ or $\mu_{R}=\mu_{F}=\xi M$, where $E_{T}^{\gamma}$ and $M$ are the final photon transverse energy and invariant mass of produced lepton pair, respectively. We vary the parameter $\xi$ between $1 / 2$ and 2 about the default value $\xi=1$ in order to estimate the scale uncertainties of our calculations. Next, following to [38], we set $m_{Z}=91.1876 \mathrm{GeV}, \Gamma_{Z}=2.4952 \mathrm{GeV}$, $\sin ^{2} \theta_{W}=0.23122$ and use the LO formula for the strong coupling constant $\alpha_{s}\left(\mu^{2}\right)$ with $n_{f}=4$ active quark flavors at $\Lambda_{\mathrm{QCD}}=200 \mathrm{MeV}$, so that $\alpha_{s}\left(m_{Z}^{2}\right)=0.1232$. Since we investigate a wide region of $E_{T}^{\gamma}$ and $M$, we use the running QED coupling constant $\alpha\left(\mu^{2}\right)$. To take into account the non-logarithmic loop corrections to the dilepton production cross

\footnotetext{
${ }^{4}$ In [30] the TMD sea quark contributions have been simulated using the off-shell gluon-gluon fusion subprocess.

${ }^{5}$ We have used the leading-order MSTW'2008 parton densities [37] as input in the KMR prescription.
} 
section we apply the effective $K$-factor, as it was done in [39]:

$$
K=\exp \left[C_{F} \frac{\alpha_{s}\left(\mu^{2}\right)}{2 \pi} \pi^{2}\right],
$$

where color factor $C_{F}=4 / 3$. A particular scale choice $\mu^{2}=\mathbf{p}_{T}^{4 / 3} M^{2 / 3}$ (with $\mathbf{p}_{T}$ being the transverse momentum of produced lepton pair) has been proposed [39] to eliminate sub-leading logarithmic terms. Note we choose this scale to evaluate the strong coupling constant in (7) only. Everywhere the multidimensional integration have been performed by the means of Monte Carlo technique, using the routine VEGAS [40]. The corresponding $\mathrm{C}++$ code is available from the authors on request 6 .

We now are in a position to present our numerical results. The ATLAS Collaboration has measured [2] the direct photon plus jet production cross sections as a function of the photon transverse energy $E_{T}^{\gamma}$, leading jet transverse momentum $p_{T}^{\text {jet }}$ and rapidity $y^{\text {jet }}$, photon-jet invariant mass $M^{\gamma \text {-jet}}$, difference $\Delta \phi^{\gamma-\text { jet }}$ between the azimuthal angles of the photon and jet and scattering angle $\cos \theta^{*}$ in the photon-jet centre-of-mass frame. In addition, the differential cross section $d \sigma / d E_{T}^{\gamma}$ has been measured [1] for three different rapidity ranges of leading jet: $\left|y^{\text {jet }}\right|<1.2,1.2<\left|y^{\text {jet }}\right|<2.8$ and $2.8<\left|y^{\text {jet }}\right|<4.4$. For each rapidity configuration the same-sign $\left(\eta^{\gamma} y^{\text {jet }}>0\right)$ and opposite-sign $\left(\eta^{\gamma} y^{\text {jet }}<0\right)$ cases have been studied separately, where $\eta^{\gamma}$ is the produced photon pseudo-rapidity. The differential cross sections of associated $Z / \gamma^{*} \rightarrow l^{+} l^{-}$and jet production were measured [3] as a function of the jet transverse momentum $p_{T}^{\text {jet }}$ and rapidity $y^{\text {jet }}$ at $66<M<116 \mathrm{GeV}$, $p_{T}^{l}>20 \mathrm{GeV},\left|\eta^{l}\right|<2.5, p_{T}^{\text {jet }}>20 \mathrm{GeV}$ and $\left|y^{\text {jet }}\right|<4.4$. These measurements were also performed as a function of the dijet invariant mass $M^{\text {jet-jet }}$ and angular separation $\Delta \phi^{\text {jet-jet }}$ between the two leading jets in events with at least two jets in the final state.

To calculate the production rates of both semi-inclusive processes under consideration we apply the procedure which has been used previously in [41 43. The produced photon or lepton pair is accompanied by a number of partons radiated due to the non-collinear parton evolution. From these several jets we choose the one carrying the largest transverse energy, and then compute the semi-inclusive production cross sections. The results of our calculations are shown in Figs. $1-4$ in comparison with the ATLAS data [1-3]. We discuss first the distributions on the leading jet rapidity. One can see that our predictions based on the KMR parton densities disagree with the data and tend to underestimate them in the central rapidity region and overestimate the data in the forward one for both processes under consideration. The observed disagreement is due to our approximation for the rapidity of partons coming from the evolution ladder which form a part of final state jets. It indicates that the full hadron-level Monte-Carlo event generator (like as, for example, CASCADE) is needed to investigate these observables?. Such evaluations are out of present short note. In Fig. 1 we see that the CCFM-based predictions agree with the ATLAS data on the $y^{\text {jet }}$ distributions within the theoretical uncertainties. The distributions on the produced photon transverse energy or jet transverse momentum agree reasonably well with the ATLAS data, as it is shown in Figs. $2-4$. The exceptions are the KMR predictions for the photon transverse energy distribution in the forward $y^{\text {jet }}$ region and both predictions for the azimuthal angle separation between the photon and jet (see Figs. 2 and 3), that are also connected with the approximation applied. However, the CCFM-based predictions agree reasonably well with the ATLAS data on the photon $p_{T}^{\gamma}$ spectrum in a whole rapidity region. The sensitivity of predicted cross sections to the TMD quark densities is clearly visible in the $\Delta \phi^{\gamma-\text { jet }}$ and $\Delta \phi^{\text {jet-jet }}$ distributions,

\footnotetext{
${ }^{6}$ lipatov@theory.sinp.msu.ru

${ }^{7}$ Very recently, the associated $W^{ \pm}+\mathrm{n}$ jets production has been studied [19] with CASCADE.
} 
as it is shown in Figs. 2 and 4. It coincides with observations of many papers (see, for example, [19, 30] and references therein). None of the TMD quark densities under consideration describe well the $\Delta \phi^{\gamma-\text { jet }}$ one at $\Delta \phi^{\gamma-\text { jet }} \sim 0$, although the CCFM-based predictions agree well with the data on $\Delta \phi^{\text {jet-jet }}$ distribution. Our calculations show that

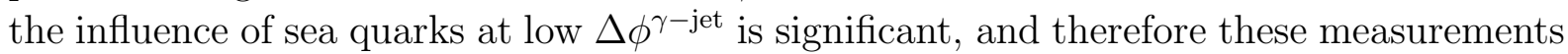
can be used to better constrain the TMD sea quark distributions.

To conclude, in the present note we have applied the TMD quark and gluon densities calculated using the formalism [25] to investigate the associated production of real or virtual photons and hadronic jets at the LHC. This study is an extension of previous one [29] where the inclusive Drell-Yan lepton pair production has been investigated. The formalism [25] is based on the TMD gluon-to-quark splitting function [26] which contains all single logarithmic small- $x$ corrections to sea quark evolution for any order of perturbation theory. Despite our approximation in description of jets, we obtained a reasonably well agreement between our predictions for the distributions of final particles on the transverse momenta and recent data [1 3] taken by the ATLAS Collaboration at the LHC. We demonstrated that studies of such processes provide an important information about the TMD parton densities in a proton at moderate and high scales, up to $\mu^{2} \sim m_{Z}^{2}$. In particular, the sensitivity of predicted cross sections to the TMD quark distributions is clearly visible in the azimuthal angle correlations between the produced photons or Drell-Yan lepton pairs and/or jets. It is important for further investigations of small- $x$ physics at hadron colliders, in particular, in the direction which concerns the non-linear effects originating from high parton densities at small $x$. However, for more detailed analysis of considered semi-inclusive processes, the full hadron-level Monte-Carlo event generator should be used.

Acknowledgements. The authors are grateful to H. Jung and S.P. Baranov for very useful discussions and comments. This research was supported by the FASI of Russian Federation (grant NS-3042.2014.2), RFBR grant 13-02-01060 and the grant of the Ministry of education and sciences of Russia (agreement 8412). We are also grateful to DESY Directorate for the support in the framework of Moscow-DESY project on Monte-Carlo implementation for HERA - LHC.

\section{References}

[1] ATLAS Collaboration, Phys. Rev. D 85, 092014 (2012).

[2] ATLAS Collaboration, Nucl. Phys. B 875, 483 (2013).

[3] ATLAS Collaboration, Phys. Rev. D 85, 032009 (2012).

[4] C.F. Berger, Z. Bern, L.J. Dixon, F. Febres Cordero, D. Forde, H. Ita, D.A. Kosower, D. Maitreet, Phys. Rev. D 78, 036003 (2008);

C.F. Berger, Z. Bern, L.J. Dixon, F. Febres Cordero, D. Forde, T. Gleisberg, H. Ita, D.A. Kosower, D. Maitre, Phys. Rev. D 82, 074002 (2010);

H. Ita, Z. Bern, L.J. Dixon, F. Febres Cordero, D.A. Kosower, D. Maitre, Phys. Rev. D 85, 031501 (2012).

[5] S. Catani, M. Fontannaz, J.Ph. Guillet, E. Pilon, JHEP 0205 028, (2002).

[6] L.V. Gribov, E.M. Levin, M.G. Ryskin, Phys. Rep. 100, 1 (1983);

E.M. Levin, M.G. Ryskin, Yu.M. Shabelsky, A.G. Shuvaev, Sov. J. Nucl. Phys. 53, 657 (1991). 
[7] S. Catani, M. Ciafoloni, F. Hautmann, Nucl. Phys. B 366, 135 (1991);

J.C. Collins, R.K. Ellis, Nucl. Phys. B 360, 3 (1991).

[8] E.A. Kuraev, L.N. Lipatov, V.S. Fadin, Sov. Phys. JETP 44, 443 (1976);

E.A. Kuraev, L.N. Lipatov, V.S. Fadin, Sov. Phys. JETP 45, 199 (1977);

I.I. Balitsky, L.N. Lipatov, Sov. J. Nucl. Phys. 28, 822 (1978).

[9] M. Ciafaloni, Nucl. Phys. B 296, 49 (1988);

S. Catani, F. Fiorani, G. Marchesini, Phys. Lett. B 234, 339 (1990);

S. Catani, F. Fiorani, G. Marchesini, Nucl. Phys. B 336, 18 (1990);

G. Marchesini, Nucl. Phys. B 445, 49 (1995).

[10] B. Andersson et al. (Small-x Collaboration), Eur. Phys. J. C 25, 77 (2002);

J. Andersen et al. (Small-x Collaboration), Eur. Phys. J. C 35, 67 (2004);

J. Andersen et al. (Small-x Collaboration), Eur. Phys. J. C 48, 53 (2006).

[11] J.C. Collins, Foundations of perturbative QCD, Cambridge University Press, 2011.

[12] E. Avsar, arXiv:1108.1181 [hep-ph]; arXiv:1203.1916 [hep-ph].

[13] F. Dominguez, C. Marquet, B.-W. Xiao, F. Yuan, Phys. Rev. D 83, 105005 (2011).

[14] S.M. Aybat, T.C. Rogers, Phys. Rev. D 83, 114042 (2011).

[15] M.G. Echevarria, A. Idilbi, A. Schäfer, I. Scimemi, Eur. Phys. J. C 73, 2636 (2013); M.G. Echevarria, A. Idilbi, I. Scimemi, Phys. Lett. B 726, 795 (2013); Phys. Rev. D 90, 014003 (2014).

[16] F. Hautmann, H. Jung, JHEP 0810, 113 (2008).

[17] M. Deak, H. Jung, K. Kutak, in Proceedings of $16^{\text {th }}$ International Workshop on DeepInelastic Scattering and Related Subjects (DIS'2008), 7 - 11 April 2008, University College London, UK; arXiv:0807.2403 [hep-ph].

[18] M. Deak, F. Hautmann, H. Jung, K. Kutak, DESY-10-179; DESY-11-262; in Proceedings of $46^{\text {th }}$ Rencontres de Moriond on QCD and High Energy Interactions, La Thuile, Italy, March 20 - 27, 2011.

[19] H. Jung, M. Krämer, A.V. Lipatov, N.P. Zotov, Phys. Rev. D 85, 034035 (2012); JHEP 1101, 085 (2011).

[20] S. Dooling, F. Hautmann, H. Jung, arXiv:1406.2994 [hep-ph].

[21] A. Gawron, J. Kwiecinski, W. Broniowski, Phys. Rev. D 68, 054001 (2003).

[22] M.A. Kimber, A.D. Martin, M.G. Ryskin, Phys. Rev. D 63, 114027 (2001); Eur. Phys. J. C 12, 655 (2000).

[23] A.D. Martin, M.G. Ryskin, G. Watt, Phys. Rev. D 70, 014012 (2004); Eur. Phys. J. C 31, 73 (2003).

[24] S. Höche, F. Krauss, T. Teubner, Eur. Phys. J. C 58, 17 (2008).

[25] S. Catani, F. Hautmann, Nucl. Phys. B 427, 475 (1994); Phys. Lett. B 315, 157 (1993).

[26] F. Hautmann, M. Hentschinski, H. Jung, Nucl. Phys. B 865, 54 (2012). 
[27] H. Jung, G.P. Salam, Eur. Phys. J. C 19, 351 (2001);

H. Jung et al., Eur. Phys. J. C 70, 1237 (2010).

[28] F. Hautmann, M. Hentschinski, H. Jung, arXiv:1207.6420 [hep-ph].

[29] S.P. Baranov, A.V. Lipatov, N.P. Zotov, Phys. Rev. D 89, 094025 (2014).

[30] S.P. Baranov, A.V. Lipatov, N.P. Zotov, Phys. Rev. D 77, 074024 (2008).

[31] A.V. Lipatov, N.P. Zotov, J. Phys. G 36, 125008 (2009).

[32] A.V. Lipatov, M.A. Malyshev, N.P. Zotov, JHEP 1112, 117 (2011).

[33] H. Jung, in Proceedings of $12^{\text {th }}$ International Workshop on Deep-Inelastic Scattering and Related Subjects (DIS'2004), Strbske Pleso, Slovakia, April 14-18, 2004; arXiv:hep-ph/0411287.

[34] G. Curci, W. Furmanski, R. Petronzio, Nucl. Phys. B 175, 27 (1980).

[35] M.A. Kimber, A.D. Martin, M.G. Ryskin, Phys. Rev. D 63, 114027 (2001);

G. Watt, A.D. Martin, M.G. Ryskin, Eur. Phys. J. C 31, 73 (2003).

[36] V.N. Gribov and L.N. Lipatov, Sov.J. Nucl. Phys. 15, 438 (1972);

L.N. Lipatov, Sov. J. Nucl. Phys. 20, 94 (1975);

G. Altarelli, G. Parisi, Nucl. Phys. B 126, 298 (1977);

Yu.L. Dokshitzer, Sov. Phys. JETP 46, 641 (1977).

[37] A.D. Martin, W.J. Stirling, R.S. Thorne, G. Watt, Eur. Phys. J. C 63, 189 (2009).

[38] C. Amsler et al. (PDG Collaboration), Phys. Lett. B 667, 1 (2008).

[39] A. Kulesza, W.J. Stirling, Nucl. Phys. B 555, 279 (1999).

[40] G.P. Lepage, J. Comput. Phys. 27, 192 (1978).

[41] A.V. Lipatov, N.P. Zotov, Phys. Rev. D 72, 054002 (2005).

[42] A.V. Lipatov, N.P. Zotov, Phys. Rev. D 81, 094027 (2010).

[43] A.V. Lipatov, M.A. Malyshev, N.P. Zotov, Phys. Rev. D 88, 074001 (2013). 

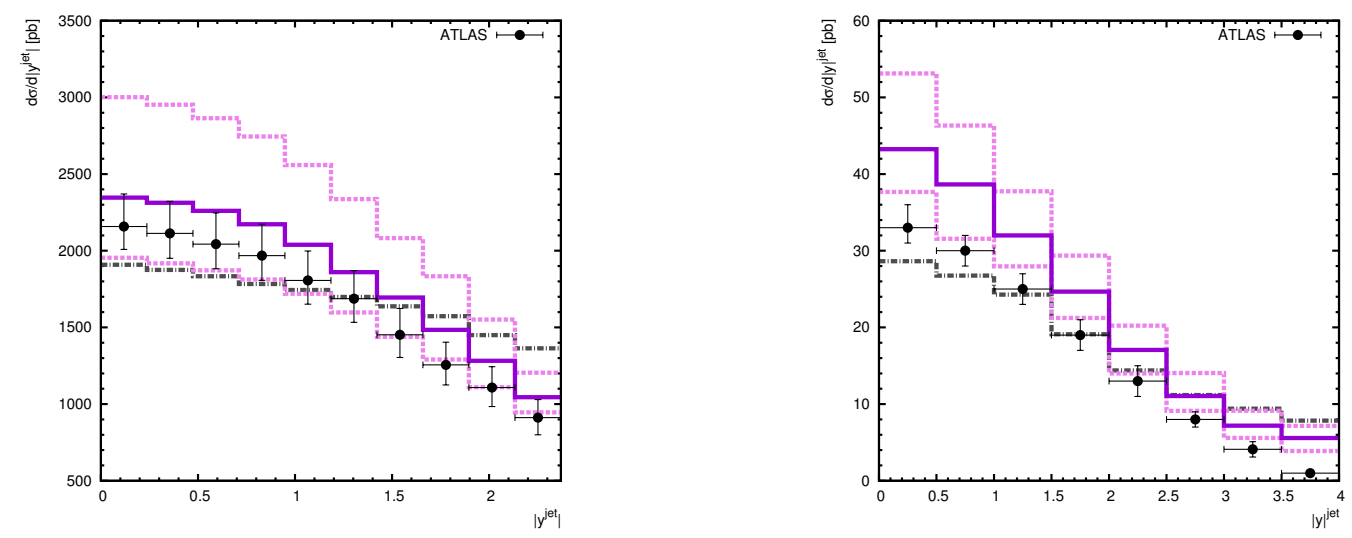

Figure 1: The differential cross sections of associated direct photon (left panel) or lepton pair (right panel) and jet production in $p p$ collisions at the LHC as a function of leading jet rapidity. The solid and dash-dotted histograms correspond to the CCFM-based and KMR predictions, respectively. The upper and lower dashed histograms correspond to the scale variations in the CCFM calculations, as it is described in the text. The experimental data are from ATLAS [2,3].
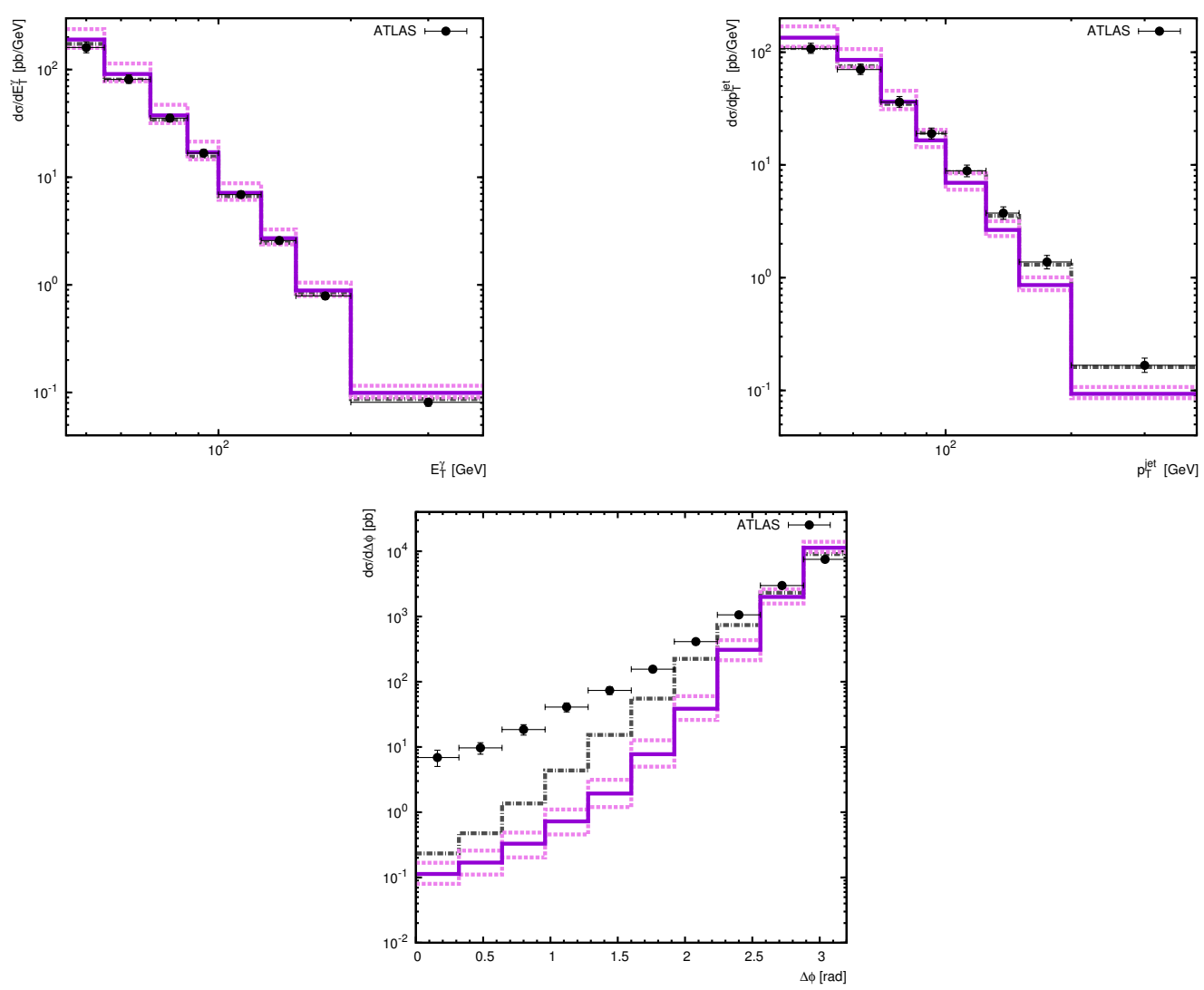

Figure 2: The differential cross sections of associated direct photon and jet production in $p p$ collisions at the LHC. Notation of histograms is the same as in Fig. 1. The experimental data are from ATLAS [2]. 

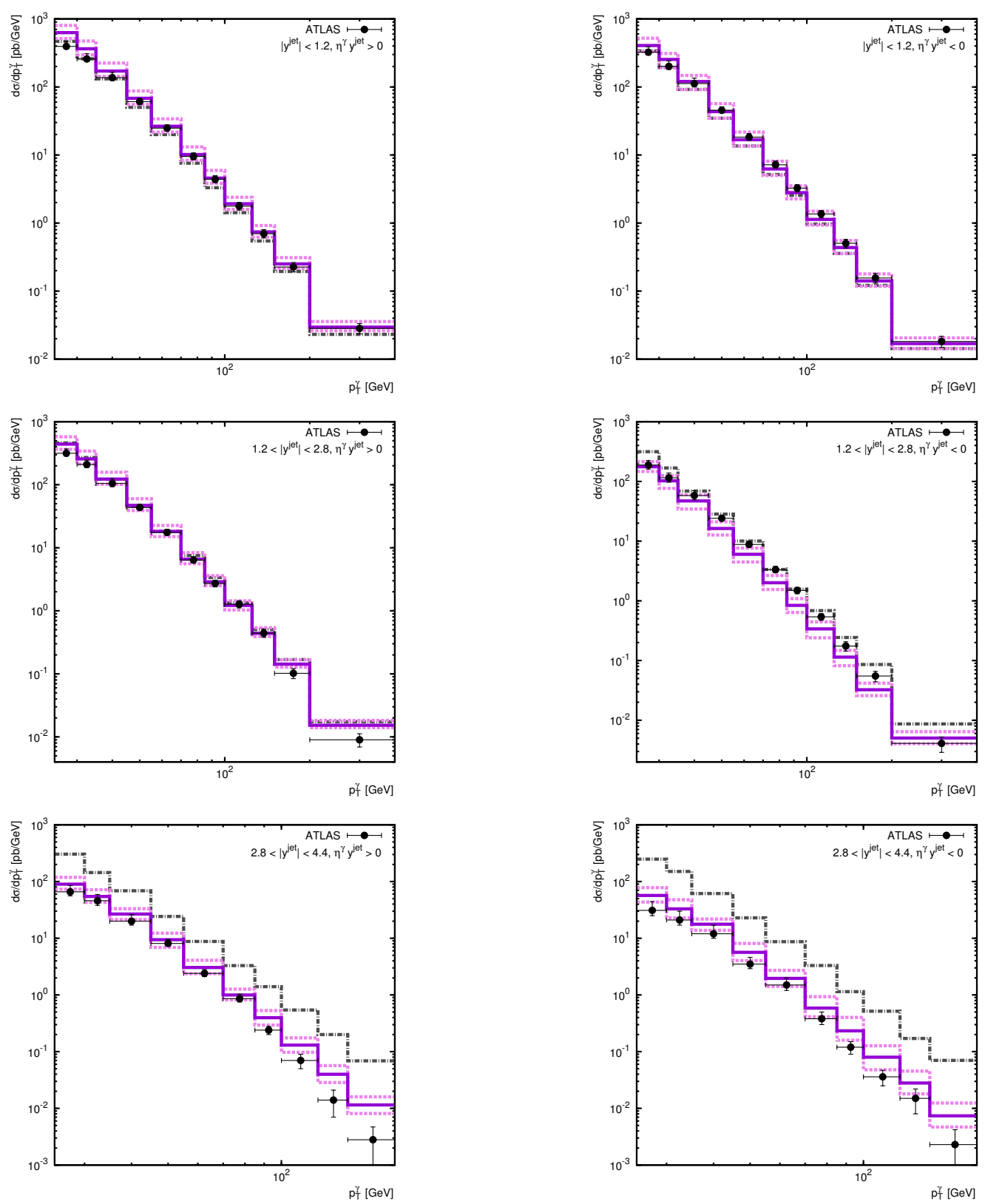

Figure 3: The differential cross sections of associated direct photon and jet production in $p p$ collisions at the LHC. Notation of histograms is the same as in Fig. 1. The experimental data are from ATLAS [1]. 

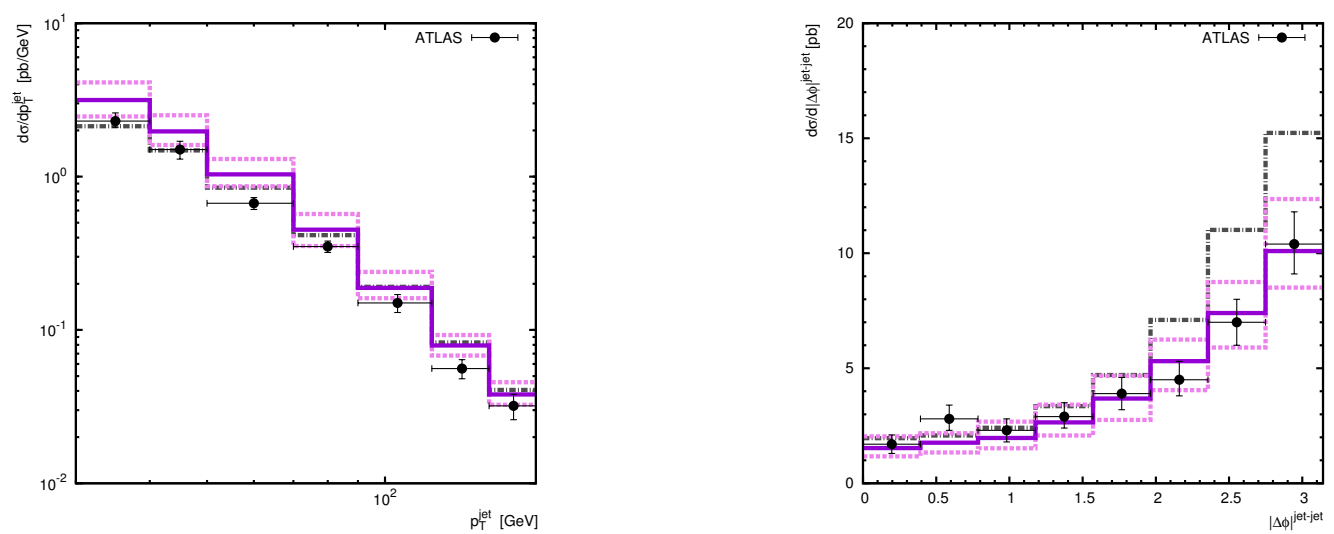

Figure 4: The differential cross sections of associated lepton pair and jet production in $p p$ collisions at the LHC. Notation of histograms is the same as in Fig. 1. The experimental data are from ATLAS [3]. 